\title{
émulations
}

\section{Jean-Pierre Dozon - La vérité est ailleurs. Complots et sorcellerie}

N’koué Emmanuel Sambieni

Émulations - Revue de sciences sociales

2019, Comptes rendus critiques, En ligne.

Article disponible à l'adresse suivante

https://ojs.uclouvain.be/index.php/emulations/article/view/crsambieni

Pour citer cet article

N’koué Emmanuel Sambieni, « Jean-Pierre Dozon - La vérité est ailleurs. Complots et sorcellerie », Émulations, en ligne. Mise en ligne le 4 août 2019.

DOI : 10.14428/emulations.cr.069

Distribution électronique : Université catholique de Louvain (Belgique) : ojs.uclouvain.be

(C) Cet article est mis à disposition selon les termes de la Licence Creative Commons Attribution, Pas d'Utilisation Commerciale 4.0 International. http://creativecommons.org/licenses/by-nc/4.0/

Éditeur : Émulations - Revue de sciences sociales / Presses universitaires de Louvain https://ojs.uclouvain.be/index.php/emulations

ISSN électronique : 1784-5734

PUL PRESSES UNIVERSITAIRES 


\title{
Jean-Pierre Dozon - La vérité est ailleurs. Complots et sorcellerie
}

\author{
N'koué Emmanuel Sambieni ${ }^{1}$
}

Recensé : Jean-Pierre Dozon, La vérité est ailleurs. Complots et sorcellerie, Paris, Maison des Sciences de l'Homme, 2017 («Interventions »), 87 p.

L'ouvrage de Jean-Pierre Dozon traite de la sorcellerie et des théories du complot dans l'espace social africain en contexte de globalisation. Il s'inscrit dans l'anthropologie sociale et culturelle, particulièrement dans l'anthropologie des croyances, du christianisme en Afrique et du changement social. La contribution essentielle de cet ouvrage est de montrer comment la sorcellerie, déjà présente dans la cosmogonie africaine, a été renforcée par le christianisme évangélique importé des États-Unis d'Amérique au moment où les institutions religieuses traditionnelles et les structures de l'économie des sociétés agraires sont en changement. La sorcellerie a par la suite servi de toile de fond pour expliquer, sur fond de rumeur parfois, les conséquences du libéralisme économique sur les individus et les familles, les représentations et la gestion politique et institutionnelle des épidémies qui se sont répandues en Afrique à partir des années 1970 (VIH et Ebola principalement). La montée des fondamentalismes religieux, notamment du christianisme, donne un grand écho à la sorcellerie, vécue et exprimée, et aux théories du complot.

L'ouvrage se compose de neuf chapitres. L'introduction pose le paradigme du double ${ }^{2}$ et du complot dans la réalité sociale vécue en Afrique. Ici, J.P. Dozon présente un imaginaire social de la dualité du réel, avec une face visible et une face invisible que même les «meilleurs esprits » (p. 8) ne peuvent pas voir. Les événements de la vie sont vus comme en continuel dédoublement, sous deux registres, l'un admettant l'administration de la preuve et l'autre non. Tout événement, entendu comme phénomène, est explicable par des preuves pour les personnes portées vers la véridicité et la plausibilité. Le même événement est explicable par une logique immatérielle pour toute personne qui pense que la cause des faits « est ailleurs » (p. 7). Cette dualité des choses constitue le fondement des théories du complot, fondement selon lequel des gens cachés manipulent les événements visibles.

\footnotetext{
${ }^{1}$ Laboratoire d'études et de recherches sur les dynamiques sociales et le développement local (Lasdel), Université de Parakou, Bénin.

${ }^{2}$ Le double est le fait, pour une réalité, d'avoir deux faces dont une qui est cachée aux gens ordinaires et une autre visible seulement pour ceux qui ont une puissance donnée.
} 
Le premier chapitre décrit et analyse la représentation de la réalité double dans une communauté ivoirienne au travers de l'expérience quotidienne de la sorcellerie. Après avoir souligné que l'Europe du $15^{\mathrm{e}}$ au $16^{\mathrm{e}}$ siècle a connu une théorie du complot autour du diable comme conspiration dans la religion, l'auteur relève que la société bété est constituée de deux scènes, à savoir celle du vis-à-vis et celle du double :

C'est cette manière de penser la réalité, ou plus précisément les événements plus ou moins inattendus qui la constituent et viennent jeter le trouble dans l'ordre des choses, qui est fréquemment mobilisée, spécialement par certains types de clairvoyants et de guérisseurs, pour expliquer les infortunes diverses, maladies et morts soudaines, aussi bien que pour expliquer des fortunes surprenantes, telle qu'une accession inopinée à une position de pouvoir ou à quelque richesse. (p. 17)

La particularité sorcellaire des Bété est doublement perçue: celle des aînés est positivement vue et celle des cadets et des pauvres est négativement qualifiée. La première reflète le pouvoir ; et la seconde est redoutée et punie.

Dans le deuxième chapitre de l'ouvrage, l'auteur présente l'appropriation de la sorcellerie en contexte de "modernité " en Afrique: changement des structures sociales et des modes d'actions quotidiens du fait de l'urbanité, de l'école, des services étatiques et des religions importées. Au-delà des croyances fortement répandues, les milieux populaires africains pensent que les événements de la vie, en l'occurrence les événements malheureux (difficulté d'emploi, problèmes de couple, chômage, adultère, etc.) passent constamment aux filtres de la sorcellerie évolutive ou contextuelle. En effet, les transformations de la Côte d'Ivoire post-coloniale, avec l'économie du cacao, ont induit des situations de fortunes et d'infortunes traduites au quotidien comme des résultats de la sorcellerie ; ce qui amène J.-P. Dozon à employer le terme de " plasticité » au regard de la figure mouvante d'une sorcellerie qui traverse dans le temps les espaces «traditionnels» villageois vers les villes ivoiriennes. Dans sa forme moderne, la sorcellerie s'est traduite par un ensemble « d'échecs et de réussites, d'enrichissements et d'appauvrissements, d'accès à des biens et à des styles de vie qui sont l'éclatante manifestation de cette modernisation (confort urbain, compte en banque, voiture, etc.), mais qui se distribuaient très inégalement au sein de la population ivoirienne» (p. 25). La percée évangélique des prophètes des Églises du réveil a bouleversé les structures traditionnelles de solidarité africaine (parenté, collectivité, etc.). Le pentecôtisme (introduit sur le continent depuis le $20^{\mathrm{e}}$ siècle) et le privé libéral (introduit de force par les programmes d'ajustement structurel avec le FMI et la Banque mondiale par exemple) ont agi sur les schèmes cognitifs de la réalité sociale (lecture double des faits), notamment la sorcellerie qui en est la plus haute expression. Il est couramment admis que seule la sorcellerie peut aider à devenir riche ou chef, tout autant que pauvre et malheureux. On note ainsi un mélange quotidien de rumeurs et de pratiques sociales, avec des procédés magiques et des sacrifices pour accéder soit au pouvoir, soit au confort matériel et social. Les mêmes rumeurs sont construites autour des pratiques de trafic des organes humains à l'échelle internationale. Elles ont été ravivées de nouveau 
dans l'interprétation des épidémies d'Ebola au regard des méthodes d'isolement des corps préconisées par les services de santé dans les stratégies de prévention.

Le troisième chapitre, Forces du mal et complotisme à l'heure de la globalisation, présente le contexte actuel de la globalisation comme producteur ou adjuvant des logiques de la sorcellerie. En effet, la globalisation est aussi favorisée par l'idéologie libérale d'origine américaine. Elle s'est développée en Afrique avec le néo-pentecôtisme qui a propagé l'esprit du diable et de la sorcellerie. Les deux mouvements (globalisation et néo-pentecôtisme) soutiennent l'idée d'une richesse individuelle qui est possible. La première la justifie par l'économie libérale et le second parle du Saint-Esprit. Les pasteurs qui se répandent partout en Afrique, en passant d'abord par le Libéria, enseignent désormais que l'individu avec la foi peut s'enrichir par le fait de l'Esprit Saint. En revanche, toujours selon les discours des pasteurs, les personnes sans foi récoltent l'infortune comme condition sociale, caractérisée par la pauvreté et les situations difficiles dont le diable est tenu pour responsable. Le néo-pentecôtisme a introduit de ce fait « une massive diabolisation du monde » (p. 34). La rhétorique du diable a occasionné des pratiques exorcistes, comme l'indique Sariette Batiboniak dans un récent ouvrage sur le pentecôtisme camerounais (2017), contre "l'univers démoniaque » de la sorcellerie que seule l'appartenance à une Église et la foi chrétienne peuvent conjurer. Le diable est devenu l'explication de l'infortune. Par exemple, les enfants de la rue de Kinshasa sont accusés de sorcellerie et deviennent les objets de la vindicte populaire. L'auteur signale que c'est depuis les positions politiques des Américains, notamment avec la guerre du Golfe et la guerre contre le régime de Saddam Hussein (en 2003) que la rhétorique du Mal à combattre a pris son essor avec des «formes locales» différentes (p. 37). L'auteur avance que ces formes locales de " diabolisme " globalisé peuvent être interprétées comme du complotisme, au même titre que les justifications de la guerre contre le régime de Saddam Hussein - qui se sont révélées mensongères - ont été interprétées comme un complot international d'officiels politique et religieux.

Le quatrième chapitre du livre, L'Afrique, le sida et l'inversion du stigmate, montre comment le VIH a d'abord permis de stigmatiser l'Afrique avant d'être interprété comme un complot de l'Occident contre le continent, notamment l'Afrique au Sud du Sahara. Par rapport à cette pandémie, l'Afrique a porté la responsabilité de son origine (transmission de l'animal à l'homme en Afrique centrale) et de son expansion (avec des taux de transmission les plus élevés). Dans le contexte des premières conséquences des programmes d'ajustement structurel sur le continent, le sida a développé chez les Africains un sentiment de pessimisme général et de discours anti-occidental. À l'opposé, l'Occident voit un monde africain inapte à se développer, avec des facteurs comme une sexualité « débridée » (p. 41) et des coutumes ancestrales supposées avoir favorisé l'émergence et l'expansion du VIH. Émerge alors l'idée d'un complot de l'Occident contre les africains, résumé par l'auteur à travers plusieurs exemples : à la demande d'un blanc, un animal a eu un rapport sexuel avec un humain et lui a 
transmis le VIH; l'absence de médicaments est voulue par l'Occident; même les guérisseurs africains inventeurs de médicaments auraient été abattus par les Occidentaux; ceux-ci auraient introduit le virus dans le préservatif supposé lutter contre la transmission de la maladie. Ces théories conspirationnistes du sida ont reçu cependant une condamnation unanime de la part de la littérature et de la sphère politique à travers la planète, y compris d'ailleurs en Occident, et notamment aux ÉtatsUnis.

Le cinquième chapitre, D'une épidémie à l'autre, ou le complotisme comme phénomène lui-même contagieux, développe la gestion locale, africaine et internationale de l'épidémie d'Ebola en Afrique de l'Ouest (Guinée Conakry, Libéria et Sierra Leone) à partir de 2014. Cette partie traite dans un premier temps de l'accent encore mis sur Ebola comme conséquence de la " primitivité » africaine (origine africaine, rapport aux animaux dans la transmission, mode de vie, etc.) et dans un second temps de la logique complotiste anti-africaine au regard des méthodes colonialistes (en référence à la médecine coloniale dans la gestion de la peste, etc.) de gestion des épidémies mises en œuvre. Comparant certaines stratégies à la traite des noirs (isolement des patients comme des esclaves) et à la sorcellerie (gestion des corps de victimes d'Ebola sans leurs parents pour leur éviter la contamination), les populations africaines, notamment dans les pays qui ont connu l'épidémie, ont conclu à un autre complot de l'Occident contre l'Afrique et les africains.

Résumant les deux dernières parties sur les épidémies du Sida et d'Ebola, l'auteur parle dans le sixième chapitre, L'inversion du stigmate en forme d'anti-occidentalisme, d'un anti-occidentalisme africain qui puise ses racines dans l'anti-colonialisme et l'anti-impérialisme, tous deux liés aux conséquences de la colonisation et des rapports postcoloniaux. Pour conclure cette partie, l'auteur évoque les mouvements salafistes musulmans, qui à la suite des pentecôtistes, rejettent l'Occident jugé promoteur de la décadence de l'homme par le rejet du modèle traditionnel de la famille (famille large) et de la prééminence de l'homme sur la femme, entre autres valeurs sociétales (du point de vue de ses fondamentalistes religieux).

Le septième chapitre, Le diable dans tous ses états, revient sur l'extension de la sorcellerie africaine primaire (authentique) aux nouvelles sphères sociales de la réalité en Afrique d'une part, celle du pentecôtisme et du néo-pentecôtisme de l'Occident à l'Afrique, avec des adaptations et des inventions locales parfois plus intenses (Ghana et Nigéria), d'autre part. Les conséquences globales du néolibéralisme étant aussi connues aussi en Occident (notamment aux États-Unis et en Angleterre), il est presque universellement admis que le diable a pris corps partout dans le monde.

Le huitième chapitre, Redoublement fictionnel et complotisme global, fait le parallèle entre les films hollywoodiens, la sorcellerie africaine et le complotisme anti-occidental. L'auteur analyse ces trois concepts sous l'angle de la fiction. La sorcellerie, avec sa duplication de la réalité (la scène du vis-à-vis et la scène du double) est déjà dans le registre fictionnel. Les films (d'épouvante, catastrophe et de suspens) en se posant sur 
la sorcellerie pour reproduire la fiction, crée une double fiction que l'auteur appelle la fiction au carré ou le redoublement. Ainsi, du dédoublement sociétal africain au redoublement global, l'Occident crée (ou renforce) l'idée du diable et complote contre l'Afrique (au travers de plusieurs infortunes sanitaires, économiques et politiques).

Le dernier chapitre, Une modernité sorcière foisonnante, fait le récapitulatif des conditions sociohistoriques qui justifient la lecture «sorcellaire » de la réalité sociale. L'auteur revient sur les chocs sociopolitiques et institutionnels qui partent de la colonisation aux épidémies pour montrer comment le diable est devenu le fait explicatif courant et simple des choses, ce diable qui est aussi l'Occident, en référence à la logique du complot.

Pour conclure, l'auteur construit une analyse diachronique des théories du complot qui «se sont créées et propagées dans le monde occidental avec l'avènement des révolutions américaine et française pour connaître leur plein essor au cours du $19^{\mathrm{e}}$ siècle, les thèses de conspiration judéo-maçonniques étant au centre de leurs fantasmagories » (p. 80). Ces théories du complot ne relèvent donc pas d'une ancienne histoire. Elles ont un lien avec le capitalisme, la techno-science et la démocratie qui en sont les principaux vecteurs.

Dans une perspective sociohistorique, l'auteur postule que la sorcellerie africaine, par sa plasticité, s'est accaparée de la modernité, une modernité non seulement africaine, mais globale pour donner sens aux déséquilibres et inquiétudes sociales conséquentes des changements sociaux. Évoquant le concept de modernité sorcière, complotiste, diabolique ou obscurantiste, l'auteur se retrouve en porte-à-faux avec le projet lumineux et éclaireur de la modernité sous l'angle de la science. C'est cette contradiction entre une modernité politique claire et une modernité sociale sombre qui lui fait avancer l'idée que la vérité est ailleurs. Accorder un nouveau crédit à la vie politique et démocratique et à l'espace publique à travers les hommes qui l'animent, cela pourrait ramener la vérité de l'ailleurs à l'ici.

Au terme de cette lecture, l'ouvrage de J.-P. Dozon est d'un grand apport scientifique et théorique en ce qui concerne l'anthropologie sociale, religieuse, de la santé et même du quotidien. Cela d'autant plus qu'il a permis de donner un sens local et global à la fois à la réalité sociale au moyen de paradigmes qui se diffusent d'un continent à l'autre, de l'Occident à l'Afrique. La perspective explicative « socio-anthropo-historique » utilisée est assez convaincante, quand on sait et qu'on partage l'idée qu'avec le temps les modèles de pensée et de pratiques sociales se diffusent inéluctablement.

Une question fondamentale qui peut se poser après la lecture de cet ouvrage reste la suivante: comment expliquer la naissance du diable dans l'imaginaire religieux (pentecôtisme, néo-pentecôtisme) occidental, notamment aux Amériques ? L'auteur ne l'aborde pas. Mais que cette pensée soit d'origine américaine et qu'elle prenne corps avec le modèle d'interprétation de la réalité sociale en Afrique ne serait pas une chose anodine. Peut-on dans cette même perspective sociohistorique envisager la piste du voyage de la pensée sorcellaire par l'entremise de l'esclavage? L'auteur ne fait pas 
expressément le lien. On pourrait aussi dire qu'en prenant le parti de ne pas donner de matériaux empiriques ici (la méthode de collecte des données est d'ailleurs absente), l'auteur, confirmé dans les sciences sociales, s'engage à coup sûr et bien évidemment dans un débat théorique osé. La posture du double de la réalité ou de la vérité à rechercher ailleurs dans les phénomènes qui apparaissent, évoquée déjà dans le titre, est à la fois révélatrice de la pensée des acteurs sociaux, "traditionnels» comme « modernes », à l'égard de leur vécu, que de celle de l'auteur à propos de la modernité. C'est la marque d'un désespoir personnel quant au projet politique sur les plans scientifique, industriel et social.

Une dernière question, à laquelle l'auteur ne donne pas de réponse approfondie, subsiste: comment comprendre que la grande partie des intellectuels africains, y compris parmi les universitaires de haute qualité, croient au diable et ont constamment peur de l'invisible ? En d'autres termes, comment comprendre que l'école, malgré ses efforts pour introduire la pensée positiviste, causale, déductive et inductive ne parvient pas à réduire l'effet des interventions pentecôtistes sur les intellectuels ? Dans les services publics, et même dans les universités de la plupart des pays d'Afrique au Sud du Sahara, l'évolution en grade se négocie dans les coulisses du spirituel, avec des prières et des sacrifices. La lecture sociale du «double » en toute chose est générale. La vérité est conçue comme toujours logée « ailleurs ». 